\title{
PREDICTION OF STUDENT PERFORMANCE USING DECISION TREE CLASSIFIER
}

\author{
V Ramakrishna Sajja ${ }^{1}$, P Jhansi Lakshmi ${ }^{2}$, DS Bhupal Naik ${ }^{3}$ \\ and Hemantha Kumar Kalluri ${ }^{4}$ \\ ${ }^{1,2,3,4}$ Department of CSE, VFSTR deemed to be University, India \\ 1vramakrishnasajja@gmail.com, ${ }^{2}$ laxmi.jhansi@gmail.com, \\ 33sbhupal@gmail.com, ${ }^{4}$ hemanth_mtech2003@yahoo.com
}

\begin{abstract}
The enormous issue of drop out students or submissive is regarding scholarly accomplishment. The academic organization wants to catch up the prompting framework while counselors be supposed to direct the arranging educational programs to their advisors. The data mining methods are very much helpful to provide the qualitative education in the academic institutions and to analyze the student performance quickly. Different classification models which can be applied in academic data mining are focused in this paper. The student problem's can be identified by applying different classification models. The goal of this paper is to enhance the performance of student and at that point anticipating the appropriated scholarly accomplishment in each major. To look at the investigation, we utilized 1200 student's data. Two measures like accuracy and error rate are assessed the framework. This method produces the $95.5 \%$ accuracy and $4.5 \%$ error rate.
\end{abstract}

Keywords - Pre processing, Classification, Decision Tree, Training, Testing, Rule Extraction, Student Performance

\section{INTRODUCTION}

Education is a major part for building up a nation. Management will look the academic achievement in part of evaluation. If the number of dropped students or resigned students is less then advisory system will be helpful to enhance the student performance. The number of resigned students can be decreased if curriculum planning in every semester has been done early. Information mining innovation can be applied in the training for enhancing an adapting, for example, the associating a learning holes, the anticipating the understudy achievement, and the finding the reasonable major. Numerous instruments are utilized in information digging for investigating an enormous dataset and discovering significant information known as learning. This study will be helpful to draw the useful conclusion. At first, the use of information mining was confined to business space yet realizes it is reached out to academics and is called as ADM.

Academic data mining (ADM) talks with data mining applications and innovative methods which examine the data at academic institutions to derive the knowledge [1]. The Knowledge Discovery Databases (KDD) derives the techniques in sight of statistics, machine learning and database systems to evaluate the big data of academic information. The interesting patterns are extracted from enormous databases by KDD process. However the competent algorithm is a key point of any application to discover the interesting patterns presented in the data considered.

Received: July 4, 2019

Reviewed: September 25, 2019

Accepted: September 30, 2019

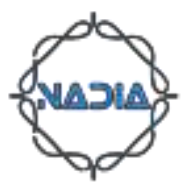


In data mining [2] and machine learning, recognizing the problem which is related among different cases based on the given training is called as classification. For example, finding the tendency of customer at Hardware shop whether he buys computer or not: "Yes" or "No". It will be decided based on the training given to the classifier across the attributes like age, student, income and credit rating etc. Decision tree classifier[3] uses a decision tree. The item value which belongs to test of attribute are represented on the branches of tree. The class labels are presented on leaf nodes of a tree. The tree pruning is one type of decision tree which improves the efficiency of classifier.

The remaining paper is ordered as follows: Literature Survey is described in Chapter II. Introduction of data mining techniques is presented in Chapter III. Methods and procedures have been discussed in Chapter IV. Practical results of experimentation are discussed in Chapter V. Chapter VI describes the conclusion of paper.

\section{LITERATURE SURVEY}

The process of discovering the knowledge from huge dataset is known as Knowledge Discovery in Databases (KDD). Academic Data Mining (ADM) is the piece of KDD that facilitate the student to enhance their academic percentage. ADM uses various other data mining techniques such as association rule discovery, classification and clustering. Several researchers felt that decision tree algorithm is reasonably good for prediction of students academic performance [4]. They executed different decision tree algorithms such as $\mathrm{J} 48$, multilayer perception, random forest, ID3, random tree and rule based with the help of WEKA tool. Split technique or cross validation technique have been used to assess the performance percentage. It is observed that student academic performance will be improved due to the usage of innovative data mining techniques like split method and cross validation method. In the examination [5], they considered the abilities of data mining inside the setting of the following academic institute and attempts to obtain fresh explicit data by connecting information handling procedures to scholarly learning of technical scholastic Institute of Athens. Every department of Athens institute utilized the decision tree algorithm to examine the student's survey .Different hands, Ahmed and his group [6] discussed that last grade of students will be predicted based on the various classification models. They have chosen the decision tree (ID3) strategy for their examination. Counting to the examination [7] of Kaur and his team, they have discussed the various classification and prediction algorithms to forecast the slow learners in academic zones. Various classification algorithms used by Kaur and his team include Naive Bayes, J48, Multi layer perception, J48 and REPTree in WEKA environment. The modern research was discovered with new outline, known as cloud-service decision tree classification for academic platform [8]. This examination considered the multi-target weight self-adjustment structure and proposed the cloud-administration classification with decision tree algorithm. At that point, their frame work is confirmed by different performance metrics.

\section{DATA MINING}

Knowledge Discovery in Database (KDD) or Data Mining (DM) is a procedure to discover examples [9] and connection from enormous informational collections with the different techniques, for example, AI, database frameworks and statistics [10, 11]. There are three procedures of information mining as the depicted after:

i. Preprocessing: set up the reasonable dataset and will organize in a helpful arrangement. By applying the following techniques, noise has to be eradicated [12] from the original dataset. The noise free dataset that have a connection, can be utilized to develop the framework. 
- Data cleaning: dispense mistake information or an immaterial dataset.

- Data integration: gather the dataset which derived by various basis.

- Data selection: pick the qualified characteristic which can be anticipated the great execution.

- Data transformation: modify the arrangement of properties that is anything but difficult to utilize or anticipate.

ii. Modeling: utilize the chose information for making a model. Model has to be trained with various cases by selection of suitable technique. Once model is trained then it has to be tested with new cases.

iii. Post processing: Derive the knowledge from the results.

\section{METHODS AND PROCEDURE}

In this examination, student problem's can be identified by applying different classification models and afterward assess the performance rate with the known metrics of accuracy and error rate. The test in this examination, we center on the data mining procedure, for example, Warehouse construction, preprocessing, classification (modeling), and rules extraction (post processing) which are portrayed underneath; Figure 1depicts the architecture of student performance prediction using Decision Tree.

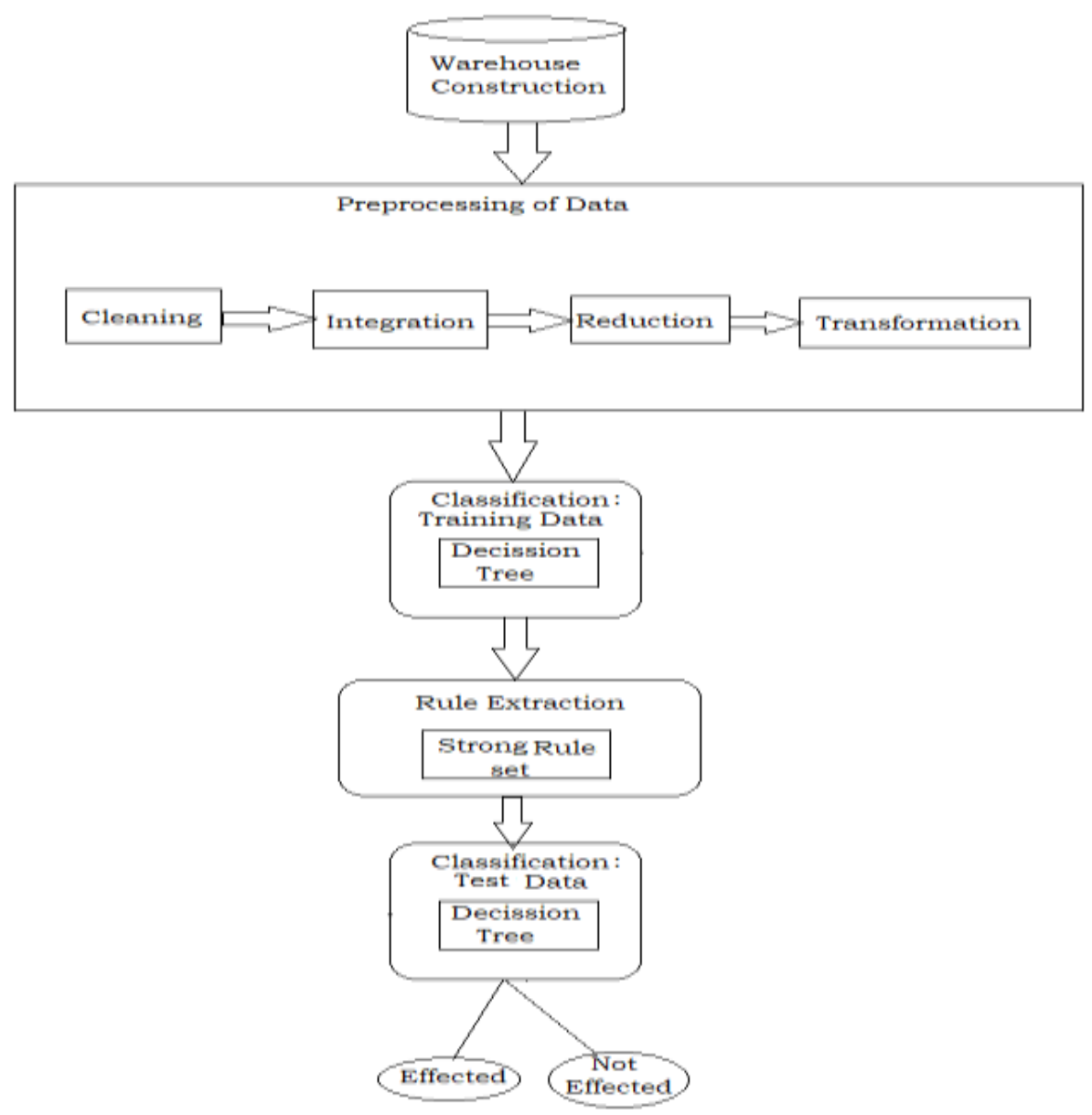

Fig. 1 Architecture of student performance prediction using Decision Tree 


\subsection{WAREHOUSE CONSTRUCTION}

In this work, a dataset of 1200 students are collected for experimentation. The total information of 1200 students is collected across 20 attributes. Types of attributes are belonging to nominal, ordinal, binary and numeric. Some of these details are collected from Vignan's Information System portal and other details which cannot retrieve from the portal are collected manually [13]. Table I describes about attributes which were used for experimentation.

Table I. Attributes Used To Collect Information of Each Student

\begin{tabular}{|c|l|c|}
\hline ID & \multicolumn{1}{|c|}{ Attribute } & Type \\
\hline A1 & Regd.No & Nominal \\
\hline A2 & Name & Nominal \\
\hline A3 & DOB & Numeric \\
\hline A4 & $10^{\text {th }}$ GP & Numeric \\
\hline A5 & Inter GP & Numeric \\
\hline A6 & Rank in Competitive exams & Numeric \\
\hline A7. & UG CGPA & Numeric \\
\hline A8 & No.of backlogs & Numeric \\
\hline A9 & Medium up to SSC & Binary \\
\hline A10 & Annual Income & Numeric \\
\hline A11 & Parent's Educational background & Ordinal \\
\hline A12 & Parent's attention & Ordinal \\
\hline A13 & Social problems & Nominal \\
\hline A14 & Behavior of student & Ordinal \\
\hline A15 & Friend circle & Binary \\
\hline A16 & No.of hours spent for academics & Numeric \\
\hline A17 & No.of hours spent for non productive work & Numeric \\
\hline A18 & Regularity of student & Binary \\
\hline A19 & Promptness on assignments & Ordinal \\
\hline A20 & Reaction towards counselor's action & Ordinal \\
\hline
\end{tabular}

\subsection{PRE PROCESSING}

Preprocessing is an important technique for any classification problems. It reduces the computational complexity of algorithm and makes the data robust and noise free. Data preprocessing includes data cleaning, data reduction, data integration and data transformation. These are explained in the following:

i. Data cleaning: It is the process of making the data as noise free [14]. During the process of data retrieval from university portal, values which were missed or wrongly loaded are filled by manual process. Some of the values can be predicted by smoothing techniques like bin means and bin boundary.

ii. Data Integration: It is the process of integrating the information which came from several sources. Tuple duplication is one of the integration techniques and this method can be used to identify duplicate tuples [15]. Duplicate tuples are identified by computing the dissimilarity matrix of mixed type for the considered dataset of 1200 records using equation 1 .

$$
\operatorname{dis}(i, j)=\frac{\sum_{a=1}^{n} \delta_{i j}^{(a)} d i s_{i j}^{(a)}}{\sum_{a=1}^{n} \delta_{i j}^{(a)}}
$$

Where 
- ' $n$ ' indicates number of attributes participated in computation.

- $\delta_{i j}^{(a)}$ is 0 if either attribute value is missing or attribute value is 0 . Otherwise 1.

- $d i s_{i j}^{(f)}$ is computed depended on attribute's type.

iii. Data reduction: It is the process of reducing the size of data. Attribute subset selection is one of the data reduction techniques and this method can be used to remove the non relevant or redundant attributes.

Forward selection and backward elimination are the two important methods of attribute subset selection. Forward selection includes the attributes to the empty set which are more relevant. Backward elimination excludes the attributes from the wholes set which are more irrelevant. Hence the attributes which would be considered for classification are $\{\mathrm{A} 4, \mathrm{~A} 5, \mathrm{~A} 6$, ,A19,A20\}.

iv. Data Transformation: It is the process of consolidating the data. Normalization is one of the data transformation techniques and this method can be used to normalize the non data by using min-max normalization.

If the values of numeric attributes are scattered in multiple ranges, those values must be in normalized form during the classification process. Hence min-max normalization is adopted to accomplish this goal. Equation 2 represents the process of min-max normalization.

$$
v_{i}^{I}=\frac{v_{i}-\min _{A}}{\max _{A}-\min _{A}}\left(\text { new_max }_{A}-\text { new_min }_{A}\right)+\text { new_min }_{A}
$$

Where

- $v_{i}$ represents the value to be normalized.

- $\min _{A}$ represents the minimum value among the all values of attribute 'A'.

- $\max _{A}$ represents the maximum value among the all values of attribute 'A'.

- $n e w_{-} \min _{A}$ represents the new minimum value considered for all values of

- attribute 'A'

- $n e w_{-} \max _{A}$ represents the new maximum value considered for all values of attribute ' $A$ '

\subsection{CLASSIFICATION}

Classification is a sort of data investigation that signifies models depicting significant data classes. Such models, called classifiers, predict class labels. A classification model can be constructed to classify bank advance applications as either safe or unsafe [16]. Such examination helps in superior comprehension of the data. Many such classification techniques have been introduced by researchers in AI, statistics, and pattern recognition. 
Classification works in two phases, which includes training and testing phases. In training phase, the classifier model is built by training data and in testing phase, a tuple with unknown class label is given to classifier as an input and extracts output with a class label.

Various classifiers can be used for classifying the data. Classifiers such as Decision Tree, Bayes, Rule based, SVM, Back propagation, ANN etc. In this paper, Decision Tree [17] is adopted to classify the student data. Figure 2 shows a sample Decision Tree classification.

4.3.1. DECISION TREE INDUCTION: Decision tree induction is the learning of decision trees from class-labeled preparing instances[18]. A decision tree is a flowchartlike tree structure, where each inward node (non leaf node) means a test on a characteristic, each branch speaks to a result of the test, and each leaf node holds a class name. The highest node in a tree is the root node. A common decision tree is appeared in Figure 2. It speaks to the idea purchases PC. It predicts whether a client at All Electronics is probable to buy a PC. Inner nodes are depicted by square shapes, and leaf nodes by ovals. Few decision tree algorithms generate binary trees, though others can create non binary trees.

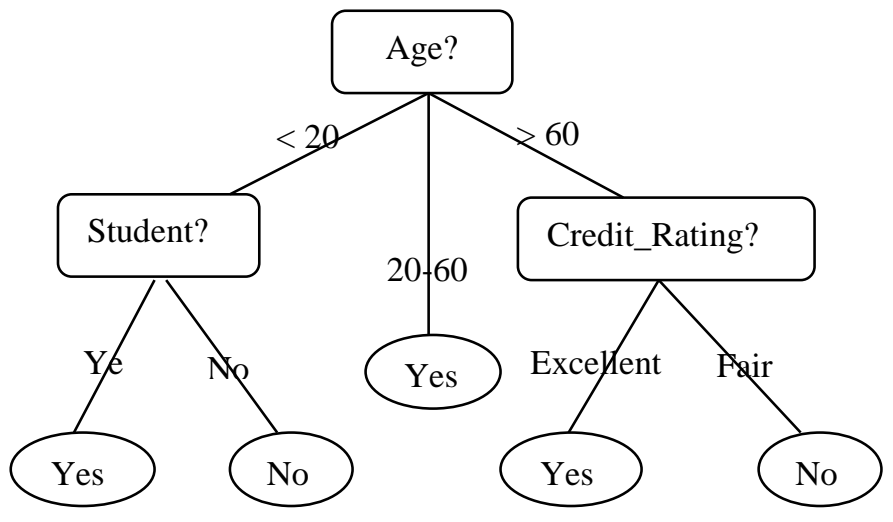

Fig. 2 Example Decision Tree

\subsubsection{DECISION TREE ALGORITHM:}

Step1: Grant all training instances as a root node of the tree. Fix the current node as a root node.

Step2: For each and every attribute

a. Split all the data instances with the help of attribute value at the node.

b. Calculate the information gain (IG) ratio from the above partition.

Step3: Identify the highest information gain (IG) ratio feature. Use this instance for partitioning process at the current node.

a. If the highestIG ratio is zero, then tag the current node as a leaf node and return.

Step4: Partition each and every instancebased on the attribute value of the best feature.

Step5: Tag each Partition as a descendant node to the current node.

Step6: For everydescendantnode:

a. If the descendantnode is "pure" denote it as a leaf node and return. 
b. If the descendantnode is not "pure"then set the descendantnode as a current node and goto step 2.

4.3.3. ATTRIBUTE SELECTION: The splitting criterion discloses to us which ascribe to test at hub $\mathrm{N}$ by deciding the "best" approach to separate or divide the tuples in $\mathrm{D}$ into individual classes. The splitting criterion likewise discloses to us which branches to develop from hub $\mathrm{N}$ concerning the results of the picked test. All the more explicitly, the splitting criterion demonstrates the splitting ascribe and may likewise show either a split-point or a split subset.

The part model is resolved so that, in a perfect world, the subsequent segments at each branch are as "pure" as would be prudent. A division is pure if all the tuples in it have a place with a similar class. At the end of the day, on the off chance that we split up the tuples in D as indicated by the fundamentally unrelated results of the part model, we trust in the subsequent segments to be as pure as would be prudent.

In this paper, A data set with 20 attributes have to be transformed as non binary tree which tells the paths from root node to leaf node that gives respective class labels. Attribute selection measure is the best splitting criterion to partition the data. Information Gain, Gain ratio and Gini Index are the attribute selection measures. One of the attribute selection measure can be adopted to split the data.

a) INFORMATION GAIN: Information gain is one of the attribute selection measure. It will be computed for all the 17 attributes considered for construction of decision tree. The attribute which gets highest information gain will be the root node in a decision tree. The same process will be repeated till reach the class label. 'D' denotes the whole dataset and 'A' denotes the respective attribute. Equation 3, 4 and 5 have been used to compute the information gain.

$$
\begin{aligned}
& \operatorname{Gain}(A)=\operatorname{Info(D)}-\operatorname{Info}_{A}(D) \\
& \operatorname{Info}(D)=-\sum_{i=1}^{m} p_{i} \log _{2}\left(p_{i}\right)
\end{aligned}
$$

Where ' $m$ ' is the number of class labels. ' $p_{i}$ ' is the probability for the $\mathrm{i}^{\text {th }}$ class label.

$$
\operatorname{Info}(D)=\sum_{j=1}^{v} \frac{\left|D_{j}\right|}{D} X \operatorname{Inf} o\left(D_{j}\right)
$$

Where ' $v^{\prime}$ is the number of values presented for the attribute 'A'. ' $D_{j}{ }^{\prime}$ is the number of tuples for the $\mathrm{j}^{\text {th }}$ value of attribute 'A' in a whole data set 'D'.

b) GAIN RATIO: GainRatio is one of the attribute selection measure. It can be taken as another measure and computed for all the 17 attributes considered for construction of decision tree. The attribute which gets highest GainRatio will be the root node in a decision tree. The same process will be repeated till reach the class label. 'D' denotes the whole dataset and 'A' denotes the respective attribute. Equation 6 and 7 have been used to compute the Gain Ratio.

$$
\operatorname{GainRatio}(A)=\frac{\operatorname{Gain}(A)}{\operatorname{SplitInfo}(D)}
$$




$$
\operatorname{SplitInfo}_{A}(D)=-\sum_{j=1}^{v} \frac{\left|D_{j}\right|}{D} X \log _{2}\left(\frac{\left|D_{j}\right|}{D}\right)
$$

Where ' $v$ ' is the number of values presented for the attribute 'A'. ' $D_{j}{ }^{\prime}$ is the number of tuples for the $\mathrm{j}^{\text {th }}$ value of attribute ' $A$ ' in a whole data set ' $\mathrm{D}$ '.

c) GINI INDEX: The Gini index splits attribute into two partitions. Assume that $A$ is a discrete-valued attribute with 'w' distinct values, $\left\{a_{1}, a_{2}, \ldots \ldots \ldots, a_{w}\right\}$, presenting in $D$. Best binary split will be determined at attribute 'A' by cross checking all the probable subsets that can be formed using known values of $A$.

For instance, if performance has three probable values, namely \{poor, average, good\} then the possible subsets are $\{$ poor, average, good $\}$, \{poor, average $\},\{$ poor, good $\}$, $\{$ average, good\}, \{poor\}, \{average $\},\{$ good $\}$, and \{\} . We exclude the power set, $\{$ poor, average, good $\}$, and the empty set from consideration since, conceptually, they do not represent a split. Therefore, there are $2^{\mathrm{v}}-2$ possible ways to form two partitions of the data, $D$, based on a binary split on $A$.

'D' denotes the whole dataset and 'A' denotes the respective attribute. Equation 8, 9 and 10 have been used to compute the Gini Index.

$$
\begin{aligned}
& \Delta \operatorname{Gini}(A)=\operatorname{Gini}(D)-\operatorname{Gini}_{A}(D) \\
& \operatorname{Gini}(D)=1-\sum_{i=1}^{m} p_{i}^{2}
\end{aligned}
$$

Where ' $m$ ' is the number of class labels. ' $p_{i}{ }^{\prime}$ is the probability for the $\mathrm{i}^{\text {th }}$ class label.

$$
\operatorname{Gini}_{A}(D)=\frac{\left|D_{1}\right|}{D} \operatorname{Gini}\left(D_{1}\right)+\frac{\left|D_{2}\right|}{D} \operatorname{Gini}\left(D_{2}\right)
$$

Where ' $D_{1}{ }^{\prime}$ is the number of tuples considered from dataset ' $\mathrm{D}$ ' with respect to attribute 'A' under first split. ' $D_{2}{ }^{\prime}$ is the number of tuples considered from dataset ' $\mathrm{D}$ ' with respect to attribute 'A' under second split.

The resultant decision tree can be viewed in Figure 3 with 69 number of leaves and 137 levels of the tree.

\subsection{RULE EXTRACTION MINING}

Rule extraction mining [19] is considered as post processing technique. Rules can be extracted from the tree also called feature extraction [20]. If ' $n$ ' number of paths are generated from root node to each leaf node called as class label then ' $n$ ' number of rules are derived. All those rules are called as strong rules. Rules include single dimensional, multi dimensional and hybrid dimensional association rules.

\section{EXPERIMENTAL RESULTS}

WEKA is a popular data mining tool which solves the many data mining problems. Dataset with 1200 records have been taken for experimentation. 1000 records are utilized for training the classifier and 200 records are used for validation or testing. Table II demonstrates the dataset and its divisions. 
Table II. Student Dataset Used For the Experimentation

\begin{tabular}{|c|c|c|c|}
\hline $\begin{array}{c}\text { Student } \\
\text { Dataset }\end{array}$ & $\begin{array}{c}\text { Good } \\
\text { students }\end{array}$ & $\begin{array}{c}\text { Poor } \\
\text { students }\end{array}$ & Total \\
\hline Training & 770 & 230 & 1000 \\
\hline Testing & 120 & 80 & 200 \\
\hline Total & 890 & 310 & 1200 \\
\hline
\end{tabular}

Results are formulated in confusion matrix. Confusion matrix has four important terms labeled as TP, TN, FP and FN and those can be used in computing many evaluation measures. Those four terms are also called as building blocks for any classifier. These terms are summarized in the confusion matrix of Table III.

Table III. Confusion Matrix

\begin{tabular}{|c|c|c|c|}
\hline CLASS & YES & NO & TOTAL \\
\hline YES & TP & FN & P \\
\hline NO & FP & TN & N \\
\hline TOTAL & $\mathrm{P}^{1}$ & $\mathrm{~N}^{1}$ & $\mathrm{P}+\mathrm{N}$ \\
\hline
\end{tabular}

TP is pronounced as true positive which refer to the positive tuples those are rightly classified. TN is pronounced as true negative which refer to the negative tuples those are rightly classified. FP is pronounced as false positive which refer to the negative tuples those are wrongly classified. FN is pronounced as false negative which refer to the positive tuples those are wrongly classified.

Accuracy of the classifier is computed using the metric accuracy which calculates the ratio of the total number of correct predictions from whole data by using the equation 11 .

$$
\text { Accuracy }=\frac{T P+T N}{T P+T N+F P+F N}
$$

Error rate is also called as misclassification rate of a classifier. It is computed as simply 1-accuracy and it is determined by using equation 12 .

$$
\text { Error Rate }=\frac{F P+F N}{T P+T N+F P+F N}
$$

After performing the experimentation on the considered dataset according to the proposed architecture, it produces $95.5 \%$ accuracy, $4.5 \%$ error rate. Resultant values for building blocks of the classifier such as TP, FP, FN and TN are shown in Table IV.

Table IV. Confusion Matrix for Testing Data

\begin{tabular}{|c|c|c|c|}
\hline CLASS & YES & NO & TOTAL \\
\hline YES & 116 & 4 & 120 \\
\hline NO & 5 & 75 & 80 \\
\hline TOTAL & 121 & 79 & 200 \\
\hline
\end{tabular}


Proposed method produces $95.5 \%$ of accuracy with $4.5 \%$ error rate over the Navie Bayes and J48 classifiers with $91.3 \%$ of accuracy with $8.7 \%$ error rate and $89.2 \%$ of accuracy with $10.8 \%$ error rate respectively. Results are shown in Table V and Figure 3.

Table V. Comparative study of different classifiers

\begin{tabular}{|c|c|c|}
\hline METHOD & ACCURACY & ERROR RATE \\
\hline Proposed & $95.5 \%$ & $4.5 \%$ \\
\hline Naive Bayes & $91.3 \%$ & $8.7 \%$ \\
\hline $\mathrm{J} 48$ & $89.2 \%$ & $10.8 \%$ \\
\hline
\end{tabular}

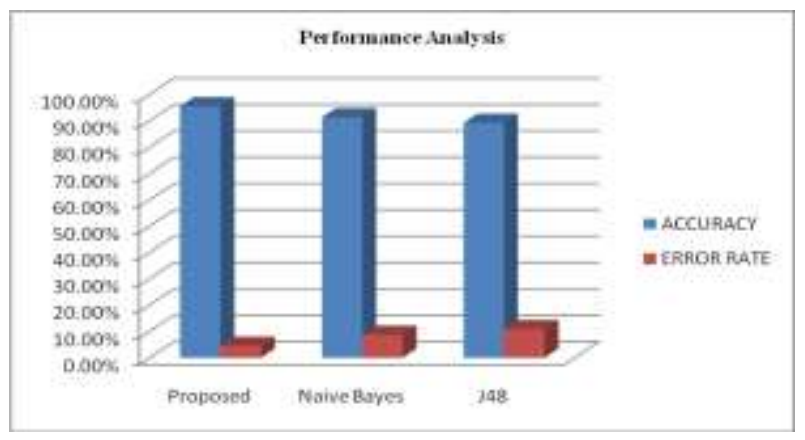

Fig. 3 Performance analysis graph

\section{CONCLUSION AND FUTURE SCOPE}

In our research, we used data mining techniques like preprocessing, classification and post processing to improve the performance of student. Raw data set is undergone through preprocessing to make ready for classification. Dataset of 1200 records are loaded and then Decision Tree is employed to classify the data. The proposed method produces accuracy of $95.5 \%$ and $4.5 \%$ error rate.

Attributes and samples considered for the experimentation can be increased to produce better results. Advanced techniques like SVM also can be employed to improve the accuracy of system.

\section{REFERENCES}

[1] M Al-Razgan, A. S. Al-Khalifa, and H. S. Al-Khalifa, "Educational data mining: A systematic review of the published literature 2006- 2013", In Proceedings the 1st International Conference on Advanced Data and Information Engineering, 2013, pp.711-719.

[2] Markus Hofmann, Ralf Klinkenberg,"RapidMiner: Data Mining Use Cases and Business Analytics Applications (Chapman and Hall/CRC Data Mining and Knowledge Discovery Series)", CRC Press, October 25, 2013.

[3] J. R. Quinlan. "Induction of Decision Trees". Mach. Learn. 1, 1 (Mar. 1986), 81-106.

[4] Kaur, Hardeep. "A Literature Review from 2011 TO 2014 on Student's Academic Performance Prediction and Analysis using Decision Tree Algorithm." Journal of Global Research in Computer Science 9.5 (2018): 10-15.

[5] Rao, NV Krishna, et al. "A Review on Data Mining Approach used in Education Data Mining using Decision Tree Algorithm.” (2018): 1735- 1738.

[6] Ahmed, Abeer Badr El Din, and Ibrahim Sayed Elaraby. "Data Mining: A prediction for Student's Performance Using Classification Method." World Journal of Computer Application and Technology 2.2 (2014): 43-47.

[7] J. Han, M. Kamber, Data Mining Concepts and Techniques, Morgan Kaufmann, 2000.

[8] B. Kami'nski, M. Jakubczyk, P. Szufel. "A framework for sensitivity analysis of decision trees". Central European Journal of Operations Research, 2017. 
[9] Muhammad Arif and Amil Roohani Dar, "Survey on Fraud Detection Techniques Using Data Mining", International Journal of $\mathrm{u}$ - and e - Service, Science and Technology, SERSC Australia, ISSN: 20054246 (Print); 2207-9718 (Online), vol. 8, no.3, March (2015), pp. 163-170, http://dx.doi.org/10.14257/ijunnesst.2015.8.3.15.

[10] "Data Mining Curriculum", ACM SIGKDD. 2006-04-30. Retrieved 2014-01-27.

[11] DS. Bhupal Naik, S. Deva Kumar, S. V. Ramakrishna, "Parallel Processing Of Enhanced K-Means Using OpenMP", 2013 IEEE International Conference on Computational Intelligence and Computing Research, 2013, pp.1-4.

[12] Kaur, Parneet, Manpreet Singh, and Gurpreet Singh Josan. "Classification and prediction based data mining algorithms to predict slow learners in education sector." Procedia Computer Science 57 (2015): 500-508.

[13] Venkatramaphanikumar S., et al., "A NOVEL PREDICTION MODEL FOR ACADEMIC EMOTIONAL PROGRESSION OF GRADUATES", ARPN Journal of Engineering and Applied Sciences, VOL. 10, NO. 6, APRIL 2015.

[14] Liu Jin-sheng, Guoxi Sun, Qinghua Zhang and He jun, "Similarity Distance Noise Reduction of Entropy Based on Lifting KNN Classification Performance", International Journal of Security and Its Applications, SERSC Australia, ISSN: 1738-9976 (Print); 2207-9629 (Online), vol.9, no.2, February (2015), pp. 149-158, http://dx.doi.org/10.14257/ijsia.2015.9.2.14.

[15] Nasrin Kalanat and Mohammad Reza Kangavari, "Data Mining Methods for Rule Designing and Rule Triggering in Active Database Systems", International Journal of Database Theory and Application, SERSC Australia, ISSN: 2005-4270 (Print); 2207-9688 (Online), vol.8, no.1, February (2015), pp. 3944, http://dx.doi.org/10.14257/ijdta.2015.8.1.05.

[16] Ji-Hoon Seo, Ho-Sun Lee and Jin-Tak Choi, "Classification Technique for Filtering Sentiment Vocabularies for the Enhancement of Accuracy of Opinion Mining", International Journal of u - and e Service, Science and Technology, SERSC Australia, ISSN: 2005-4246 (Print); 2207-9718 (Online), vol. 8, no.10, October (2015), pp. 11-20, http://dx.doi.org/10.14257/ijunnesst.2015.8.10.02.

[17] Chao, Wang, and Wang Junzheng. "Cloud-service decision tree classification for education platform." Cognitive Systems Research 52 (2018): 234-239.

[18] Bhanu Prakash Battula, KVSS Rama Krishna, Tai-hoon Kim, "An Efficient Approach for Knowledge Discovery in Decision Trees using Inter Quartile Range Transform", International Journal of Control and Automation, SERSC Australia, ISSN: 2005-4297 (Print); 2207-6387 (Online), vol. 8, no. 7, July (2015), pp. 325-334, http://dx.doi.org/10.14257/ijca.2015.8.7.32.

[19] Rokhmat Rokhmat, Yunus Indra Purnama, Eka Novita Sari and Tutut Herawan, "Applying Dependency of Attributes for Business Data Mining in Information Systems: A Theoretical Framework", International Journal of Control and Automation, SERSC Australia, ISSN: 2005-4297 (Print); 22076387 (Online), vol. 8, no. 4, April (2015), pp. 241-256, http://dx.doi.org/10.14257/ijca.2015.8.4.24.

[20] Shiliang Zhang and Tingcheng Chang, "A Hybrid Stock Selection Model Based on Forecasting, Classification and Feature Selection", International Journal of $\mathrm{u}$ - and e - Service, Science and Technology, SERSC Australia, ISSN: 2005-4246 (Print); 2207-9718 (Online), vol. 9, no.6, June (2016), pp. 153-168, http://dx.doi.org/10.14257/ijunnesst.2016.9.6.15. 
International Journal of Advanced Science and Technology Vol.131 (2019) 